Published in final edited form as:

Cardiovasc Revasc Med. 2019 July ; 20(7): 546-552. doi:10.1016/j.carrev.2019.04.005.

\title{
Racial/Ethnic Disparities in Patients Undergoing Transcatheter Aortic Valve Replacement: Insights from the Healthcare Cost and Utilization Project's National Inpatient Sample
}

\author{
Dagmar F. Hernandez-Suarez, MD, MSc ${ }^{*}, \mathrm{a}$, Sagar Ranka, MD ${ }^{\mathrm{b}}$, Pedro Villablanca, MD, MSc ${ }^{\mathrm{c}}$, \\ Nicole Yordan-Lopez, MD $^{d}$, Lorena González-Sepúlveda, MSc $^{e}$, Jose Wiley, MD, MPH ${ }^{f}$, \\ Cristina Sanina, MD ${ }^{f}$, Abiel Roche-Lima, PhD ${ }^{g}$, Brenda G. Nieves-Rodriguez, MSc ${ }^{g}$, Stacey \\ Thomas, MD $^{h}$, Pedro Cox-Alomar, MD, MPH', Angel Lopez-Candales, MD, FACC ${ }^{a}$, Harish \\ Ramakrishna, MD, FESCh \\ aDivision of Cardiovascular Medicine, Department of Medicine, University of Puerto Rico School \\ of Medicine, San Juan, PR, USA. \\ bDepartment of Medicine, John H Stroger Hospital of Cook County, Chicago, IL, USA. \\ 'Division of Cardiovascular Medicine, Department of Medicine, Henry Ford Hospital, Detroit, MI, \\ USA. \\ ${ }^{\mathrm{d} D e p a r t m e n t}$ of Surgery, Kendall Regional Medical Center, Miami, FL, USA. \\ ePuerto Rico Clinical and Translational Research Consortium (PRCTRC), University of Puerto \\ Rico, San Juan, PR, USA. \\ fDivision of Cardiology, Department of Medicine, Montefiore Medical Center/Albert Einstein \\ College of Medicine, New York, NY, USA. \\ 9Center for Collaborative Research in Health Disparities, University of Puerto Rico School of \\ Medicine, San Juan, PR, USA. \\ hDivision of Cardiovascular and Thoracic Anesthesiology, Mayo Clinic, Phoenix, AZ, USA. \\ 'Division of Cardiology, Department of Medicine, Louisiana State University, New Orleans, LA, \\ USA.
}

\begin{abstract}
Purpose: To identify racial/ethnic disparities in utilization rates, in-hospital outcomes and health care resource use among Non-Hispanic Whites (NHW), African Americans (AA) and Hispanics undergoing transcatheter aortic valve replacement (TAVR) in the United States (US).
\end{abstract}

\footnotetext{
*Corresponding author: Dagmar F Hernandez-Suarez, MD. Division of Cardiovascular Medicine, School of Medicine, University of Puerto Rico Medical Sciences Campus. PO Box 365067. San Juan, Puerto Rico 00936-5067. Tel: (787)758-2525; Fax: (787)754-1739, ORCID ID: 0000-0003-1850-907, dagmar.hernandez@upr.edu.

Publisher's Disclaimer: This is a PDF file of an unedited manuscript that has been accepted for publication. As a service to our customers we are providing this early version of the manuscript. The manuscript will undergo copyediting, typesetting, and review of the resulting proofbefore it is published in its final citable form. Please note that during the productionprocess errors may be discovered which could affect the content, and all legal disclaimersthat apply to the journal pertain.

Conflict of Interest

The authors declare no conflict of interest.
} 
Methods and Results: The National Inpatient Sample database was queried for patients $\geq 18$ years of age who underwent TAVR from 2012 to 2014. The primary outcome was all-cause in hospital mortality. A total of 36,270 individuals were included in the study. The number of TAVR performed per million population increased in all study groups over the three years [38.8 to 103.8 (NHW); 9.1 to 26.4 (AA) and 9.4 to 18.2 (Hispanics)]. The overall in-hospital mortality was $4.2 \%$ for the entire cohort. Race/ethnicity showed no association with in-hospital mortality ( $\mathrm{P}>.05)$. Though no significant difference were found between AA and NHW in any secondary outcome, being Hispanic was associated with higher incidence of acute myocardial infarction ( $\mathrm{aOR}=2.02$; 95\%CI, 1.06-3.85; $\mathrm{P}=.03)$, stroke/transient ischemic attack (aOR=1.81; 95\% CI, 1.04-3.14; $\mathrm{P}=$. $04)$, acute kidney injury (aOR=1.65; 95\% CI, 1.23-2.21; $\mathrm{P}<.01)$, prolonged length of stay $(\mathrm{aOR}=1.18 ; 95 \% \mathrm{CI}, 1.08-1.29 ; \mathrm{P}<.01)$ and higher hospital costs $(\mathrm{aOR}=1.27$; 95\%CI, 1.18-1.36; $\mathrm{P}<.01)$.

Conclusion: There are significant racial disparities in patients undergoing TAVR in the US. Though in-hospital mortality was not associated with race/ethnicity, Hispanic patients had less TAVR utilization, higher in-hospital complications, prolonged length of stay and increased hospital costs.

\section{Keywords}

Transcatheter aortic valve replacement; disparity; comparative outcomes

\section{Introduction}

Transcatheter aortic valve replacement (TAVR) has recently become the mainstay of treatment for many patients with severe aortic stenosis (AS) that otherwise are considered at an inordinate risk to undergo surgical replacement [1]. Nonetheless, following the historical trend of other novel advances in the cardiovascular field, TAVR clinical studies have been mainly focused on Caucasian populations with limited representation of minority groups [2,3]. Previous studies have shown that along with African Americans (AA), Hispanics are a target of significant cardiovascular disparities and burdened with a higher prevalence of cardiovascular disease than their Caucasian counterparts [4].

With over 58,000 patients in the United States (US) undergoing TAVR as an alternative to conventional surgical aortic valve replacement (SAVR) as of 2016 [3], it is crucial to examine current utilization trends with regards to TAVR use.

Recently published studies addressing racial disparities in patients undergoing aortic valve replacement have mainly focused on the AA population [3,5]. However, little is known about TAVR use in Hispanics, the largest minority group in the United States [6]. To answer this important question, we reviewed the Nationwide Inpatient Sample (NIS) database to identify if any potential racial differences exist with regards to clinical risk profiles, TAVR utilization rates, in-hospital outcomes, resource utilization, and cost of care between NonHispanic Whites (NHW), AA, and Hispanics. 


\section{Materials and Methods}

Data was obtained from the 2012-2014 National Inpatient Sample (NIS) database files. The NIS is a publicly available de-identified database of hospital inpatient stays, sponsored by the Agency for Healthcare Research and Quality as part of the Healthcare Cost and Utilization Project [7]. It is the largest all-payer inpatient care database in the US and approximates a $20 \%$ stratified sample of all discharges from community hospitals in the 46 participating states. Discharge weights are provided for each record and can be used to obtain national estimates. Each record represents a hospital stay and includes information on primary and secondary discharge diagnoses and procedures, demographics, hospital characteristics, expected payment source, total charges, discharge status, length of stay (LOS), and comorbidity measures. A category 4 exemption was granted by the local Institutional Review Board (Protocol B0560118) of the University of Puerto Rico Medical Sciences Campus (UPR MSC) to conduct the study. The International Classification of Diseases, Ninth Edition, Clinical Modification (ICD-9-CM) procedure codes was used to identify all patients aged $\geq 18$ years undergoing TAVR (procedures codes 35.05 and 35.06) from January 2012 to December 2014 (N=41,025) (Fig. 1). Records with missing data on race, in-hospital mortality, age or gender were excluded from analysis. The final study population $(\mathrm{N}=36,270)$ was divided into 3 groups according to race/ethnicity: NHW $(\mathrm{n}=33,355)$, African-American $(\mathrm{n}=1,480)$ and Hispanics $(\mathrm{n}=1,435)$. Other races and/or ethnic groups were also excluded from our analysis. A list of ICD-9-CM codes used for selection of the study population is provided in Table S1 in the Data Supplement.

The following baseline characteristics were used in our study: demographics (age, gender, primary expected payer, median household income for patient's ZIP code, discharge disposition) and relevant comorbidities [smoking, underweight, dyslipidemia, known coronary artery disease (CAD), history of prior myocardial infarction (MI), percutaneous coronary intervention (PCI), coronary artery bypass grafting (CABG), transient ischemic attack (TIA)/stroke, atrial fibrillation, carotid artery disease, permanent pacemaker (PPM) implantation, implantable cardioverter defibrillator (ICDf) and dementia]. Other Elixhauser's comorbidities like heart failure, diabetes mellitus, hypertension, obesity, peripheral vascular disease, renal failure, anemia, chronic pulmonary disease, coagulopathy, hypothyroidism, liver disease, fluid and electrolyte disorders, cancer, pulmonary circulation disorder, alcohol abuse, depression, drug-abuse, valvular disease, psychoses and neurological disorders were also identified [8].

Hospital-level characteristics were bed size, hospital location, teaching status and census region. A list of ICD-9-CM and Clinical Classification Software codes used to identify comorbidities is included in Table S1 in the Data Supplement.

Our primary outcome of interest was all-cause in-hospital mortality. Secondary outcomes of interest were TIA/stroke, acute myocardial infarction (AMI), cardiogenic shock, cardiac arrest, major bleeding, vascular complications, new requirement for PPM, conversion to SAVR, AKI and sepsis. Major adverse cardiovascular events (MACE) and net-adverse cardiovascular events (NACE) were composite outcomes which included all-cause inhospital mortality, AMI, stroke/TIA with the latter also including major bleeding and 
vascular complications. Other evaluated secondary outcomes were length of stay (LOS) and total hospital costs for patients surviving the hospitalization. Definitions and ICD-9-CM codes for these outcomes have been used in previous studies and are enlisted in Table S1 in the Data Supplement $[9,10]$. TAVR utilization rates were calculated based on number of TAVR procedures adjusted for the total population of patients of a particular race/ethnicity for each year. National population estimates for both the overall sample and older patients ( $\geq 75$ years) were obtained from the US Census Bureau [11].

National Inpatient Sample discharge weights were applied to all statistical analyses, as recommended by the Agency for Healthcare Research and Quality [12], in order to obtain national estimates on each outcome assessed in our study. Patients' baseline and hospital characteristics, and TAVR approach (i.e., transapical or endovascular) were assessed overall and according to racial group using descriptive statistics. Differences between these groups were assessed on all these variables using Pearson Chi-squared test and adjusted Wald test, as appropriate. Unadjusted and adjusted odds ratios (ORs) were estimated using logistic regression and multinomial regression models to determine the magnitude of differences between racial groups for in-hospital patient outcomes with two (i.e., binary) or more categories, respectively. In addition, the magnitude of association (unadjusted and adjusted ORs) between race/ethnicity and hospital cost as well as patients' length of stay were estimated through a Generalized Linear Regression models with negative binomial distributions. For all these regression models, the NHW were used as reference. Models that were adjusted included the following covariates: age, gender, known coronary artery disease, prior percutaneous coronary intervention, prior coronary artery bypass graft, diabetes mellitus, renal failure, coagulopathy, fluid and electrolyte disorder, coronary heart failure, hypertension, pulmonary circulation disorder, depression, and cancer. The crude rates of patients undergoing TAVR (per million) were estimated by year and racial group. Crude rates' annual percent changes (APC) were calculated using Joinpoint Regression Models [13] to assess trends of TAVR use among each studied group. Furthermore, patients' 30-days survival was assessed by race/ethnicity thru Kaplan Meier and a Cox regression model; proportional hazard assumption test was performed. Statistical analyses were performed using Stata v.15 (College Station, Texas 77845 USA); P-values less than 0.05 were considered statistically significant.

\section{Results}

A total of 36,270 TAVR were included in our study, which represent the $88.4 \%$ of all TAVR performed in the US between 2012-2014. Non-Hispanic Whites accounted for the $92.0 \%$ of all procedures followed by AA (4.1\%) and Hispanics (3.9\%). Compared to NHW, the minority subgroups were signficantly younger (Table 1). Gender stratification of minorities showed the AA women were a majority (60.8\%) within their race/ethnicity whereas men were more represented in the Hispanic cohort (55.7\%). Comorbidity burden was distributed hetergenously. However, the AA and Hispanic cohorts were less likely to have known coronary artery disease, history of prior PCI, prior CABG and Atrial Fibrillation. Compared to minorities, NHW were less likely to have diabetes mellitus, renal failure, anemia and coagulopathies. No inter-racial differences were noted in cardiovascular comorbidities such as heart failure, peripheral vascular disease, smoking, hypertension, dyslipidemia, history of 
prior myocardial infarction, prior stroke/TIA, vavular heart disease and carotid artery disease. Also, no differences were observed in prior history of intracardiac devices placement like ICD/pacemaker between all these groups. Of notice, Hispanics had the highest proportion of patients diagnosed with Diabetes Mellitus (47.0\%) while AA (21.0\%) were more obese. Compared to NHW, AA and Hispanic populations had higher burden of Elixhauser comorbidities: $4.3 \%$ (NHW) vs $5.1 \%$ (AA) vs $4.7 \%$ (Hispanics).

In terms of median household income, both AA and Hispanics had the higher proportion of patients below the $50^{\text {th }}$ percentile. Medicare beneficiaries comprised the majority of TAVR patients across all races/ethnicities with most of them seeking care at large urban nonteaching hospitals. Geographically, about one-third of TAVR procedures were performed in the southern US with AA predominance. Similarly, Hispanics comprised $\sim 45 \%$ of cases in the western US with NHW distributed evenly across the country. About one-third of patients were discharged home postprocedure with no inter-race/ethnic differences. In addition, although most patients underwent TAVR using an endovascular approach in the three study subgroups, the transapical approach was more oftenly used in Hispanics. A $~ 2.5$ fold increase in the number of TAVR procedures done on an annual basis was observed between 2012 to 2014 (Supplement Tables S2,S3). TAVR performed per million population increased from 38.8 to 103.8 in NHW, 9.1 to 26.4 in AA, and 9.4 to 18.2 in Hispanics. Of notice, both AA and Hispanics had the lowest rates of TAVR procedures among the three study groups (Fig. 2A). In fact, for older patients ( $\geq 75$ years), TAVR utilization in Hispanics showed no significant annual percentage change (34.6\% P>.05) (Fig. 2B).

The overall in-hospital mortality was $4.2 \%$ for the entire cohort. In-hospital mortality was similar between NHW, AA and Hispanics across the study years (Table 2) with overall mortality of $4.2 \%$ in NHW, 3.7\% in AA and 5.2\% in Hispanics. Still after adjusting for demographic characteristics and TAVR access site, no significant differences were observed among these groups from 2012-2014. The same results were reproduced in a Cox's regression model where race/ethnicity showed no association with in-hospital mortality at 30-days of stay (Fig. 3).

The in-hospital mortality for endovascular and transapical TAVR was $4.0 \%$ and $5.2 \%$ respectively in the overall group. There was no significant differences in absolute in-hospital mortality among the three race/ethnic groups in either transapical or endovascular cohorts (Table S4). However, higher mortality trends were observed in minority subgroups for patients undergoing transapical TAVR: NHW $4.9 \%$ vs AA 9.5\% vs Hispanics 7.4\%.

Comparison of multiple secondary outcomes between the study subgroups showed a higher incidence of all secondary outcomes in the minority subgroups compared to NHW (Table 3). Though no statistically signicant difference were found between AA and NHW in any secondary outcome, being Hispanic was associated with higher incidence of AMI $(\mathrm{aOR}=2.02 ;$ 95\% CI $1.06-3.85, \mathrm{P}=.03)$, stroke/TIA (aOR=1.81; 95\%CI, 1.04-3.14; $\mathrm{P}=.04)$, AKI (aOR=1.65; 95\%CI, 1.23-2.21; $\mathrm{P}<.01)$, sepsis (aOR=2.34; 95\%CI, 1.30-4.22; $\mathrm{P}<.01)$, prolongued length of stay $(\mathrm{aOR}=1.18 ; 95 \% \mathrm{CI}, 1.08-1.29 ; \mathrm{P}<.01)$ and higher hospital costs $(\mathrm{aOR}=1.27 ; 95 \% \mathrm{CI}, 1.18-1.36 ; \mathrm{P}<.01)$ during hospitalization for TAVR. 


\section{Discussion}

The paucity of data on racial disparities in patients undergoing TAVR along with the limited representation of minority populations in TAVR clinical trials, prompted our study to determine the effect of race/ethnicity on TAVR utilization and in-hospital outcomes and in these patients. To the best of our knowledge, this is the largest reported study comparing racial differences for in-hospital outcomes in TAVR patients that has included the Hispanic population. Our main findings are as follows: 1) Hispanic patients had less TAVR performed than both NHW and AA with no significant increase in utilization from 2012-2014; 2) No inter-race/ethnic differences were observed for in-hospital mortality for patients undergoing TAVR; and 3) Hispanic patients had higher incidence of adverse outcomes after TAVR as compared to NHW.

Since racial/ethnic disparities have been previously reported in referrals for aortic valve replacement [14,15], it was expected that patients from racial/ethnic minority groups had less TAVR utilization. Certainly, we found that both AA and Hispanics had less proportion of TAVR performed when compared to NHW. Given that both AA and Hispanics were younger than NHW, it could be argued that they were more likely to be offered SAVR instead. However, an analysis of TAVR utilization in older patients ( $\geq 75$ years) showed the same tendency that for the overall population. In fact, it seemed that older Hispanic patients have not benefited from the increasing trend of TAVR procedures from 2012 to 2014 (APC: $64.4 \%$ in NHW vs. $51.6 \%$ in AA vs. $34.6 \%$ in Hispanics; $\mathrm{P}<.05$ ). This is of great concern given that Hispanics represent the largest racial/ethnic minority group in the US and have a high burden of cardiovascular disease [16, 17].

Although the true prevalence of severe AS in different races or ethnic groups for the study years is unknown, some trends have been previously reported using the NIS database [18]. For the first year included in our study (2012), Beydoun et al., described that compared to NHW, both AA and Hispanics had a lower prevalence of AS (2.62\% vs $1.37 \%$ and $1.73 \%$ respectively). Prevalence variations among these groups could account for the differences observed in TAVR utilization in our study. However, we observed changes in TAVR utilization rates among NHW and both AA and Hispanics that were higher than 70\% (Fig. 2A). The prior reported differences in AS prevalence [18] are not high enough to justify such a significant disproportion in TAVR utilization among NHW and Hispanics. It could therefore be suggested that these differences represent a true racial disparity in TAVR utilization. Nevertheless, more data on severe AS prevalence by race/ethnicity needs to be obtained to better reveal the nature of these disparities.

Our observed in-hospital mortality was comparable to overall predicted mortality in STS/ACC TVT TM registry from 2012-2015 [19]. In our study, though the minority groups had significantly different comorbidity burden, this was not reflected in the unadjusted or adjusted race/ethnic-specific mortality rates. Report of the STS/TVT registry shows a gradual but significant reduction in trend of in-hospital mortality, also reflected (but not compared) in our study. Thus, this suggests that the decrease in mortality might stem from procedure optimization and expertise more than the patient-related factors (age, race, etc.). 
A recent analysis of 17,973 Medicare patients undergoing TAVR showed no difference in 30-day or 1- or 2-year mortality or between Black or Hispanic compared to NHW [20]. This was in contrast to the findings of SAVR in the same study, where higher mortality was noted in Black patients. Higher mortality in minorities has been well reported in patients undergoing surgical procedures [21]. Thus, previous studies have shown race to be an independent risk factor after non-valvular cardiac surgeries which is not translated as straightforward in the surgical- or catheter-related valvular procedures [22]. It remains unclear if this difference is due to physicians, hospitals or selection bias.

Minha et al., in a single center study of 469 patients undergoing TAVR showed similar mortality and periprocedural outcomes for Black patients compared to Caucasians [2]. Despite lower incidence of severe AS in Black patients [15], they have been shown to have lower chance of being referred for SAVR despite similar severity of aortic disease and comorbidity profile. Reasons for discrepancy in the referral was either due to patient declining the procedure [23] or being lost to follow up [24]. Our study confirms the similar inter-racial periprocedural outcomes, and thus patients should be referred earlier with proper education to help improve cardiovascular care for minorities. Hispanics have been previously identified to suffer worse peri-procedural outcomes when seeking care for cardiac- or non-cardiac issues [25], especially if undergoing low- to high risk surgical procedures [26]. Along with AA, Hispanics with AS have also less access to advanced cardiac procedures, cardiac referrals and follow up appointments [3]. Though some studies $[19,20]$ and our data shows that the number of TAVR procedures are comparable for Hispanics and AA, no previous study has reported significant disparities of periprocedural TAVR outcomes, length of stay and hospital costs for former separately. Thus, this study is currently the sole evidence/datapoint adding clinically relevant information in TAVR outcomes for an important minority group in the US. Interestingly, the controversial Hispanic Paradox [27] does not appear to extend to TAVR patients, as it also failed to hold true for postoperative outcomes after surgery [26]. Based on our results, it remains unclear if any of the observed disparities might be the result of socioeconomic inequalities, inherent biases in healthcare provision, issues with regards to referrals to specialists, or language as well as cultural barriers that limit the acceptance of such a procedure by Hispanics.

Though the strength of this project lies on the sample size as well as "real world" population-based information in relation to race/ethnicity, some limitations need to be addressed. First, information on Society of Thoracic Surgeons (STS) scores and key prognostic data, as well as cause of death and long-term mortality, are not available in the NIS database. Hence, we cannot determine the impact of STS score on adverse cardiovascular outcomes and survival after hospital discharge. Second, due to the large number of variables involved in the database, there is a potential for coding errors which could bias our study. However, miscoding of diagnosis, dates of medical interventions, tests, or drug dispensing is unlikely due to their clinical and financial relevance if we consider the impact of a correct coding on claimed charges. Finally, given the observational design of our study, residual measured, or unmeasured confounding might account for some of our findings. 


\section{Conclusion}

In this analysis of the NIS database from 2012-2014, important racial disparities were observed among patients undergoing TAVR. Hispanics were the racial group with less TAVR performed during the study period. Though inhospital mortality was not associated with race/ethnicity; Hispanic patients had higher rates of in-hospital complications, prolonged length of stay and increased hospital costs. Further studies are now warranted not only to better delineate these observed racial differences; but also, to advance our understanding in terms of the underlying pathophysiological causes and treatment strategies to reduce these racial/ethnic disparities in TAVR patients.

\section{Supplementary Material}

Refer to Web version on PubMed Central for supplementary material.

\section{Acknowledgments}

This study was funded by the National Institute of Health (NIH) Award Numbers U54MD007587, S21MD001830, R25MD007607 and TL1TR001434-3. Its contents are solely the responsibility of the authors and do not necessarily represent the official views of the National Institutes of Health.

\section{References}

1. Nishimura RA, Otto CM, Bonow RO, et al. 2017 AHA/ACC Focused Update of the 2014 AHA/ACC Guideline for the Management of Patients With Valvular Heart Disease: A Report of the American College of Cardiology/American Heart Association Task Force on Clinical Practice Guidelines. J Am Coll Cardiol. 2017;70(2):252-289. [PubMed: 28315732]

2. Minha S, Barbash IM, Magalhaes MA, et al. Outcome comparison of African-American and Caucasian patients with severe aortic stenosis subjected to transcatheter aortic valve replacement: a single-center experience. Catheter Cardiovasc Interv. 2015;85(4):640-7. [PubMed: 24782407]

3. Bob-Manuel T, Sharma A, Nanda A, Ardeshna D, Skelton WP 4th, Khouzam RN. A review of racial disparities in transcatheter aortic valve replacement (TAVR): accessibility, referrals and implantation. Ann Transl Med. 2018;6(1):10. [PubMed: 29404356]

4. Davidson JA, Kannel WB, Lopez-Candales A, et al. Avoiding the looming Latino/Hispanic cardiovascular health crisis: a call to action. Ethn Dis. 2007;17(3):568-73. [PubMed: 17985515]

5. Alqahtani F, Aljohani S, Almustafa A, et al. Comparative outcomes of transcatheter aortic valve replacement in African American and Caucasian patients with severe aortic stenosis. Catheter Cardiovasc Interv. 2018;91(5):932-937. [PubMed: 28941139]

6. US Census Bureau. 2016 Population estimates. https://www.census.gov/newsroom/facts-forfeatures/2017/hispanic-heritage.html. Accessed August 27, 2018.

7. Overview of the National (Nationwide) Inpatient Sample (NIS). https://www.hcup-us.ahrq.gov/ nisoverview.jsp. Accessed February 28, 2018.

8. Elixhauser A, Steiner C, Harris DR, Coffey RM. Comorbidity measures for use with administrative data. Med Care. 1998; 36:8-27. [PubMed: 9431328]

9. Gupta T, Goel K, Kolte D, et al. Association of Chronic Kidney Disease With In-Hospital Outcomes of Transcatheter Aortic Valve Replacement. JACC Cardiovasc Interv. 2017;10(20):2050-2060. [PubMed: 29050621]

10. Gupta T, Khera S, Kolte D, et al. Transcatheter Versus Surgical Aortic Valve Replacement in Patients With Prior Coronary Artery Bypass Grafting: Trends in Utilization and PropensityMatched Analysis of In-Hospital Outcomes. Circ Cardiovasc Interv. 2018;11(4):e006179. [PubMed: 29643130] 
11. Khera R, Angraal S, Couch T, et al. Adherence to methodological standards in research using the National Inpatient Sample. JAMA. 2017;318:2011-2018 [PubMed: 29183077]

12. United States Census Bureau. https://www.census.gov. Accessed August 27, 2018.

13. National Cancer Institute. Joinpoint Trend Analysis Software. Surveillance Research Program. https://surveillance.cancer.gov/joinpoint/. Accessed September 20, 2018.

14. Cruz Rodriguez B, Acharya P, Salazar-Fields C, Horne A Jr. Comparison of Frequency of Referral to Cardiothoracic Surgery for Aortic Valve Disease in Blacks, Hispanics, and Whites. Am J Cardiol. 2017;120(3):450-455. [PubMed: 28583680]

15. Patel DK, Green KD, Fudim M, Harrell FE, Wang TJ, Robbins MA. Racial Differences in the Prevalence of Severe Aortic Stenosis. J Am Heart Assoc. 2014;3(3):e000879. [PubMed: 24870936]

16. Vespa J, Armstrong DM, Medina L. Demographic Turning Points for the United States: Population Projections for 2020 to 2060 Current Population Reports, P25-1144. Washington, DC: US Census Bureau; 2018.

17. Davidson JA, Kannel WB, Lopez-Candales A, et al. Avoiding the looming Latino/Hispanic cardiovascular health crisis: a call to action. Ethn Dis. 2007;17(3):568-73. [PubMed: 17985515]

18. Beydoun HA, Beydoun MA, Liang H, Dore GA, Shaked D, Zonderman AB. Sex, Race, and Socioeconomic Disparities in Patients With Aortic Stenosis (from a Nationwide Inpatient Sample). Am J Cardiol. 2016;118(6):860-865. [PubMed: 27481471]

19. Grover FL, Vemulapalli S, Carroll JD, et al. 2016 Annual Report of The Society of Thoracic Surgeons/American College of Cardiology Transcatheter Valve Therapy Registry. J Am Coll Cardiol. 2017;69(10):1215-1230. [PubMed: 27956264]

20. McNeely C, Zajarias A, Fohtung R, et al. Racial Comparisons of the Outcomes of Transcatheter and Surgical Aortic Valve Implantation Using the Medicare Database. Am J Cardiol. 2018;122(3): 440-445. [PubMed: 30201109]

21. Lucas FL, Stukel TA, Morris AM, Siewers AE, Birkmeyer JD. Race and Surgical Mortality in the United States. Ann Surg. 2006;243(2):281-6. [PubMed: 16432363]

22. Taylor NE, O'Brien S, Edwards FH, Peterson ED, Bridges CR. Relationship between race and mortality and morbidity after valve replacement surgery. Circulation. 2005;111(10):1305-12. [PubMed: 15769773]

23. Yeung M, Kerrigan J, Sodhi S, et al. Racial differences in rates of aortic valve replacement in patients with severe aortic stenosis. Am J Cardiol. 2013;112(7):991-5. [PubMed: 23791013]

24. Sleder A, Tackett S, Cerasale M, et al. Socioeconomic and Racial Disparities: a Case-Control Study of Patients Receiving Transcatheter Aortic Valve Replacement for Severe Aortic Stenosis. J Racial Ethn Health Disparities. 2017;4(6):1189-1194. [PubMed: 28039604]

25. Khariton Y, Nassif ME, Thomas L, et al. Health Status Disparities by Sex, Race/Ethnicity, and Socioeconomic Status in Outpatients With Heart Failure. JACC Heart Fail. 2018;6(6):465-473. [PubMed: 29852931]

26. Eguia E, Cobb AN, Kirshenbaum EJ, Afshar M, Kuo PC. Racial and Ethnic Postoperative Outcomes After Surgery: The Hispanic Paradox. J Surg Res. 2018;232:88-93. [PubMed: 30463790]

27. Markides KS, Coreil J. The health of Hispanics in the southwestern United States: an epidemiologic paradox. Public Health Rep. 1986;101(3):253-65. [PubMed: 3086917] 


\section{Highlights}

- Transcatheter aortic valve replacement (TAVR) clinical studies have been mainly focused on Caucasian populations with limited representation of minority groups.

- $\quad$ Little is known about TAVR use in Hispanics, the largest minority group in the United States.

- Hispanic patients had less TAVR utilization, higher in-hospital complications, prolonged length of stay and increased hospital costs. 


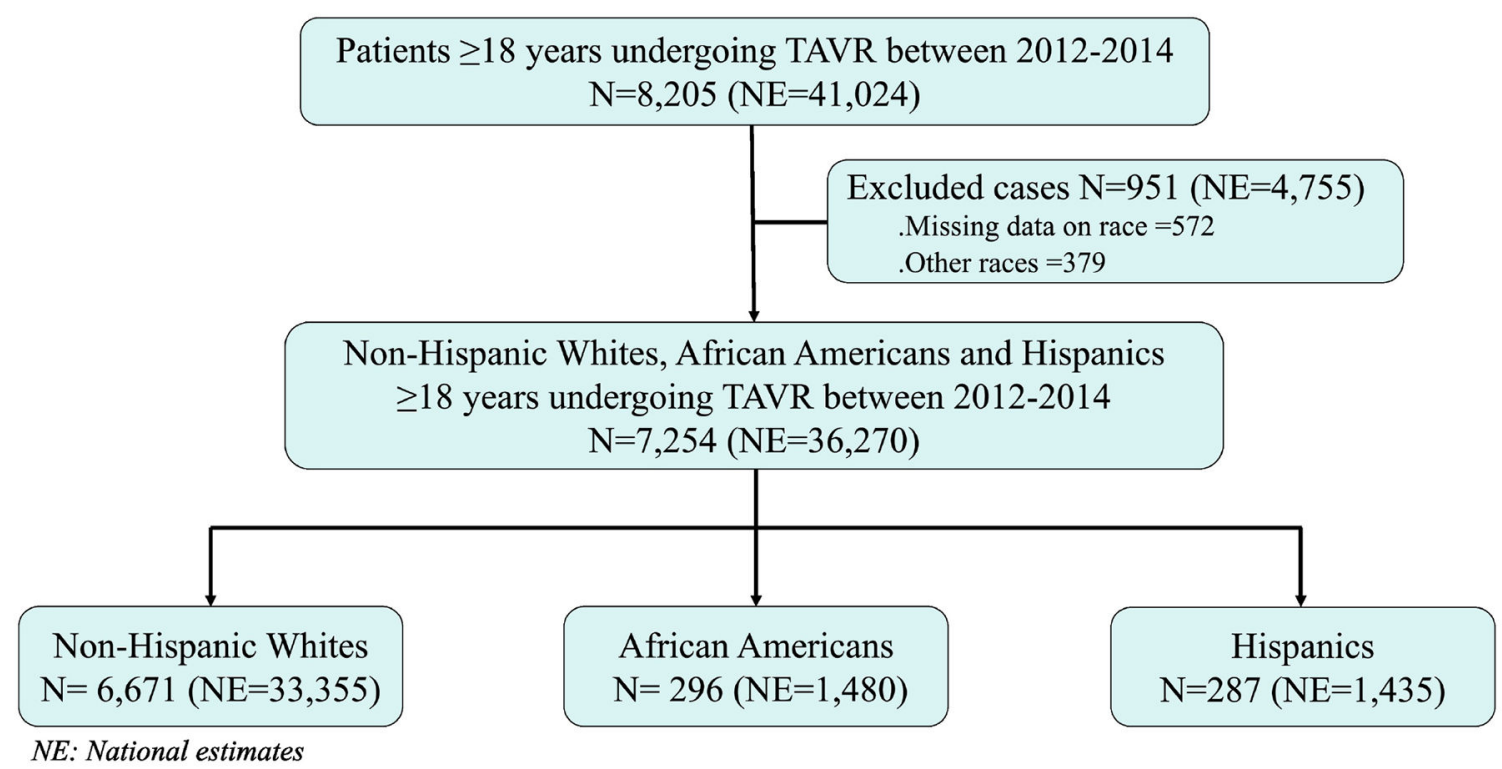

Figure 1.

Flowchart for selection of the study sample. 

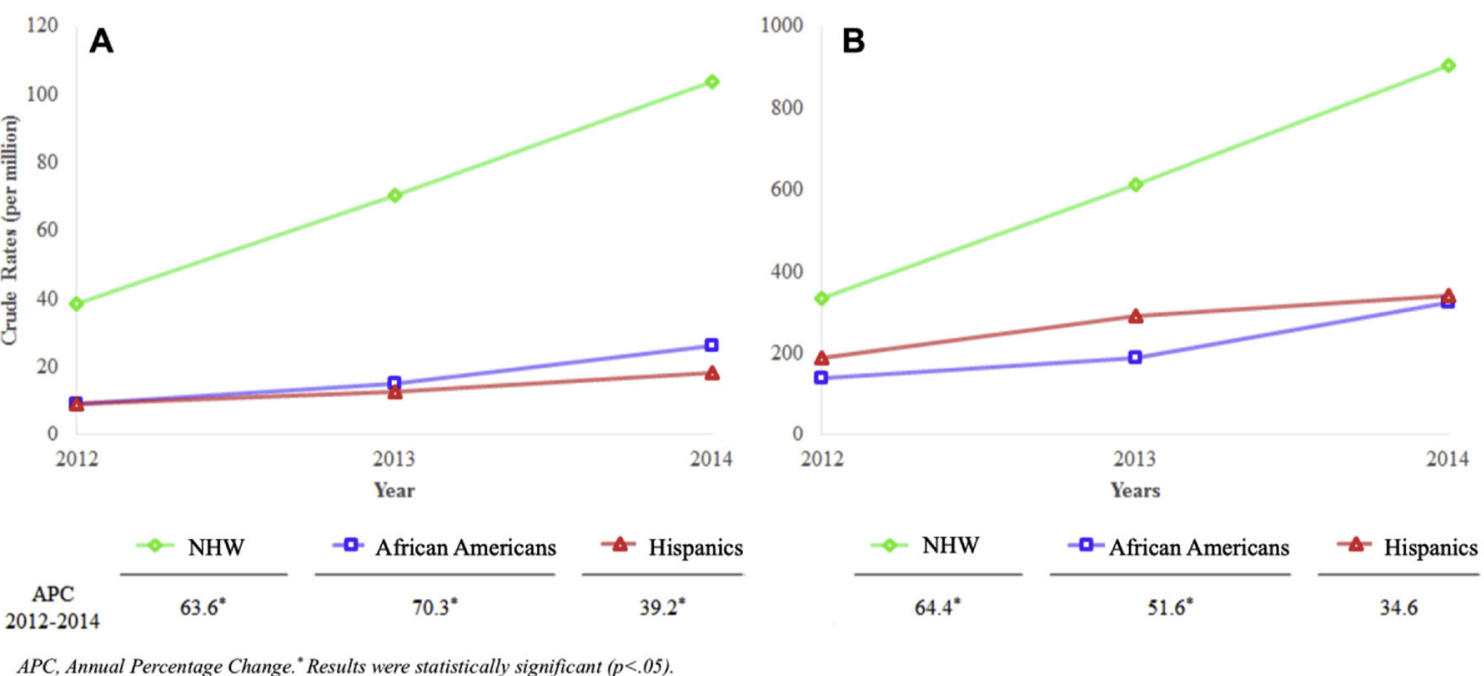

Figure 2.

Race/Ethnic variation in Utilization of TAVR in population aged: A) $\geq 18$ years, and B) $\geq 75$ years. 


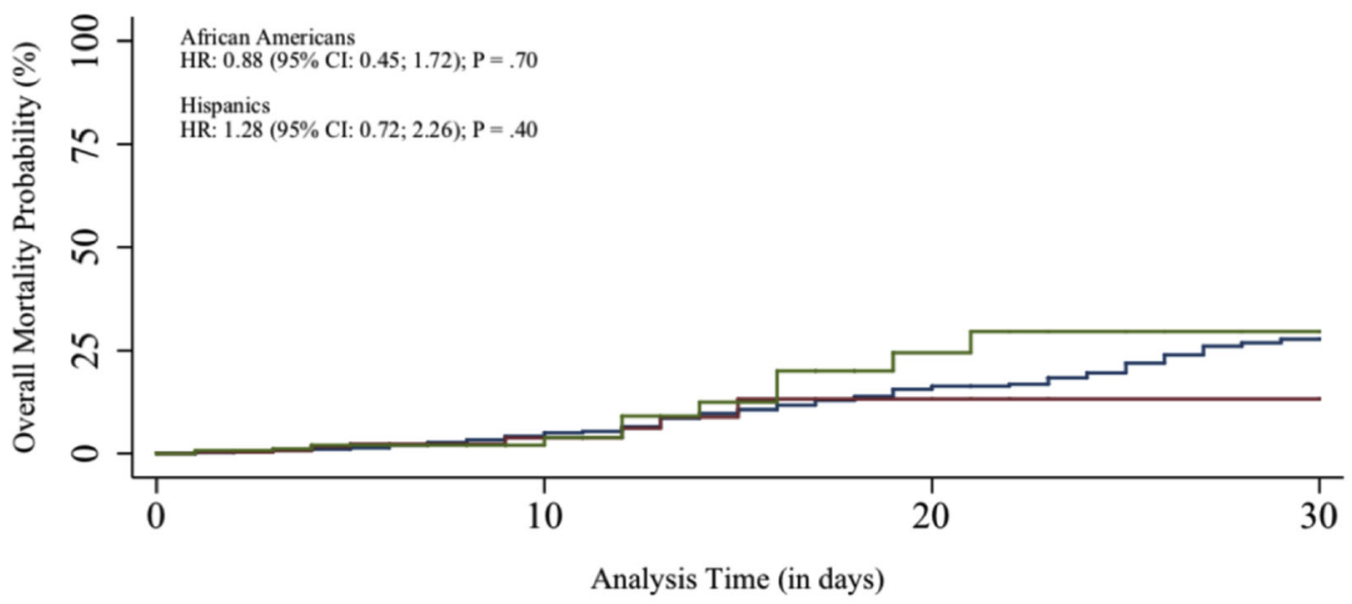

\section{Number at risk}

NHW 31790

4590

1100

385

African Americans 1440

290

75

25

Hispanics 1395

265

75

25

\begin{tabular}{lll}
\hline NHW & African Americans $\quad$ Hispanics
\end{tabular}

Figure 3.

Survival analysis of in-hospital mortality by race/ethnicity in patients undergoing TAVR. 
Table 1.

Baseline and hospital characteristics of TAVR patients.

\begin{tabular}{|c|c|c|c|c|c|}
\hline Variables & $\begin{array}{c}\text { Overall } \\
\mathbf{n}=\mathbf{3 6 , 2 7 0}\end{array}$ & $\begin{array}{l}\text { Non-Hispanic Whites } \\
\quad \mathbf{n}=\mathbf{3 3 , 3 5 5}\end{array}$ & $\begin{array}{l}\text { African Americans } \\
\quad \mathrm{n}=\mathbf{1 , 4 8 0}\end{array}$ & $\begin{array}{l}\text { Hispanics } \\
\mathrm{n}=\mathbf{1 , 4 3 5}\end{array}$ & P-value $e^{\dagger}$ \\
\hline Age (in years), $M e a n \pm S D$ & $81.1 \pm 8.5$ & $81.3 \pm 8.2$ & $77.7 \pm 10.8$ & $78.8 \pm 10.9$ & $<.001$ \\
\hline Women, \% & 47.9 & 47.4 & 60.8 & 44.3 & $<.001$ \\
\hline Smoker, \% & 27.9 & 28.3 & 23.3 & 23.7 & .05 \\
\hline Underweight, \% & 0.9 & 0.9 & 1.0 & 0.7 & .93 \\
\hline Dyslipidemia, \% & 64.6 & 64.7 & 61.2 & 64.5 & .45 \\
\hline Known CAD, \% & 71.2 & 71.7 & 61.5 & 70.0 & $<.01$ \\
\hline Prior MI, \% & 13.0 & 13.2 & 10.5 & 10.1 & .12 \\
\hline Prior PCI, \% & 18.5 & 18.8 & 12.8 & 17.4 & .04 \\
\hline Prior $\mathrm{CABG}, \%$ & 22.2 & 22.9 & 7.4 & 22.0 & $<.001$ \\
\hline Prior TIA/stroke, $\%$ & 12.6 & 12.7 & 13.9 & 9.8 & .32 \\
\hline Atrial Fibrillation, $\%$ & 44.8 & 46.2 & 25.0 & 32.4 & $<.001$ \\
\hline Carotid Artery Disease, $\%$ & 7.3 & 7.5 & 4.7 & 5.9 & .17 \\
\hline Prior PPM, \% & 10.4 & 10.5 & 7.1 & 12.2 & .10 \\
\hline Prior ICD, $\%$ & 3.0 & 3.1 & 2.0 & 1.4 & .17 \\
\hline Dementia, $\%$ & 5.0 & 5.0 & 7.4 & 3.1 & .07 \\
\hline \multicolumn{6}{|l|}{ Elixhauser comorbidities, \% } \\
\hline Heart Failure & 12.2 & 12.1 & 14.9 & 11.5 & .35 \\
\hline Diabetes Mellitus & 34.4 & 33.4 & 43.9 & 47.0 & $<.001$ \\
\hline Hypertension & 79.6 & 79.4 & 82.4 & 81.9 & .28 \\
\hline Obesity & 14.0 & 13.7 & 21.0 & 13.6 & $<.01$ \\
\hline Peripherovascular disease & 29.2 & 29.1 & 29.7 & 30.3 & .89 \\
\hline Renal failure & 35.2 & 34.3 & 51.0 & 40.1 & $<.001$ \\
\hline Anemia & 26.0 & 25.4 & 37.5 & 30.0 & $<.001$ \\
\hline Chronic Pulmonary Disease & 33.4 & 33.8 & 28.7 & 31.0 & .11 \\
\hline Coagulopathy & 23.8 & 23.4 & 27.7 & 30.0 & .01 \\
\hline Hypothyroidism & 20.4 & 20.8 & 14.5 & 18.1 & .02 \\
\hline Liver disease & 2.4 & 2.4 & 3.4 & 2.8 & .49 \\
\hline Fluid and electrolyte disorder & 25.9 & 25.6 & 32.1 & 27.5 & .04 \\
\hline Cancer & 3.6 & 3.7 & 3.4 & 3.1 & .87 \\
\hline Neurologic disorder & 6.3 & 6.4 & 5.7 & 4.9 & .54 \\
\hline Psychoses & 1.7 & 1.7 & 2.4 & 1.7 & .71 \\
\hline PUD w/o bleeding & 0.03 & 0.03 & 0.0 & 0.0 & .93 \\
\hline Valvular disease & 3.4 & 3.3 & 5.1 & 3.8 & .21 \\
\hline Pulmonary circulation disorder & 3.9 & 3.8 & 4.7 & 3.8 & .75 \\
\hline Alcohol abuse & 1.2 & 1.2 & 1.0 & 0.7 & .72 \\
\hline Rheumatoid arthritis or collagen disease & 5.1 & 5.2 & 5.7 & 3.1 & .34 \\
\hline Depression & 7.2 & 7.4 & 4.7 & 6.6 & .28 \\
\hline Drug abuse & 0.3 & 0.3 & 0.3 & 0.4 & .99 \\
\hline Mean no. of Elixhauser comorbidities, Mean $\pm S D$ & $4.3 \pm 2.2$ & $4.3 \pm 2.2$ & $5.1 \pm 2.5$ & $4.7 \pm 2.2$ & $<.001$ \\
\hline
\end{tabular}




\begin{tabular}{|c|c|c|c|c|c|}
\hline Variables & $\begin{array}{c}\text { Overall } \\
\mathbf{n}=\mathbf{3 6 , 2 7 0}\end{array}$ & $\begin{array}{l}\text { Non-Hispanic Whites } \\
\mathbf{n}=\mathbf{3 3 , 3 5 5}\end{array}$ & $\begin{array}{l}\text { African Americans } \\
\quad \mathrm{n}=\mathbf{1 , 4 8 0}\end{array}$ & $\begin{array}{l}\text { Hispanics } \\
\mathrm{n}=\mathbf{1 , 4 3 5}\end{array}$ & P-value ${ }^{\dagger}$ \\
\hline \multicolumn{6}{|l|}{ Hospital characteristics } \\
\hline Primary payer, \% & & & & & $<.001$ \\
\hline Medicare & 90.2 & 90.9 & 83.3 & 82.2 & \\
\hline Medicaid & 1.0 & 0.7 & 3.1 & 5.9 & \\
\hline Private insurance & 7.1 & 6.8 & 11.9 & 9.4 & \\
\hline Self-pay & 0.5 & 0.5 & 1.4 & 0.7 & \\
\hline Other & 1.1 & 1.1 & 0.3 & 1.8 & \\
\hline Median household income (percentiles), $\%$ & & & & & $<.001$ \\
\hline O to $25^{\text {th }}$ & 21.6 & 19.8 & 48.6 & 34.4 & \\
\hline $26^{\text {th }}$ to $50^{\text {th }}$ & 25.0 & 25.3 & 19.7 & 22.1 & \\
\hline $51^{\text {th }}$ to $75^{\text {th }}$ & 25.1 & 25.5 & 17.7 & 23.9 & \\
\hline $76^{\text {th }}$ to $100^{t h}$ & 28.3 & 29.4 & 14.0 & 19.6 & \\
\hline Bed size, \% & & & & & .50 \\
\hline Small & 4.7 & 4.9 & 2.0 & 4.2 & \\
\hline Medium & 17.2 & 17.2 & 17.2 & 16.4 & \\
\hline Large & 78.1 & 77.9 & 80.7 & 79.4 & \\
\hline Hospital location, \% & & & & & .17 \\
\hline Urban non-teaching & 88.5 & 88.2 & 91.9 & 92.0 & \\
\hline Urban teaching & 10.7 & 10.9 & 8.1 & 8.0 & \\
\hline Rural & 0.8 & 0.9 & 0.0 & 0.0 & \\
\hline Hospital region, $\%$ & & & & & $<.001$ \\
\hline Northeast & 26.1 & 26.8 & 21.6 & 15.0 & \\
\hline Midwest & 19.0 & 19.5 & 21.6 & 3.5 & \\
\hline South & 36.5 & 36.0 & 46.6 & 37.3 & \\
\hline West & 18.4 & 17.7 & 10.1 & 44.3 & \\
\hline Discharge disposition, \% & & & & & .64 \\
\hline Routine & 33.6 & 33.4 & 36.5 & 36.2 & \\
\hline Transfer - Short term hospital & 0.8 & 0.8 & 0.7 & 1.7 & \\
\hline Transfer - Other facility & 29.2 & 29.5 & 26.7 & 24.4 & \\
\hline Home health care & 32.1 & 32.1 & 32.4 & 32.4 & \\
\hline Against medical advice & 0.01 & 0.02 & 0.0 & 0.0 & \\
\hline Other & 4.2 & 4.2 & 3.7 & 5.2 & \\
\hline TAVR access site, $\%$ & & & & & $<.001$ \\
\hline Transapical & 19.7 & 19.6 & 14.2 & 28.2 & \\
\hline Endovascular & 80.5 & 80.6 & 85.8 & 71.8 & \\
\hline
\end{tabular}

Abbreviations: CAD, Coronary Artery Disease; MI, Myocardial Infarction; PCI, Percutaneous Coronary Intervention; CABG, Coronary Artery Bypass Graft; TIA, Transient Ischemic Attack; PPM, Permanent Pacemaker; ICD, Implantable Cardioverter Defibrillator; PUD, Peptic Ulcer Disease.

${ }^{\dagger}$ Adjusted Wald Test and Pearson Chi-squared test were used to calculate p-values as appropriate. 
Table 2.

Odds ratios for in hospital mortality among patients undergoing TAVR, by year and overall, according to race/ ethnicity.

\begin{tabular}{|c|c|c|c|c|c|}
\hline Years & $\begin{array}{l}\text { Non-Hispanic Whites } \\
\quad \mathbf{n}=\mathbf{3 3 , 3 5 5}\end{array}$ & $\begin{array}{c}\text { African Americans } \\
n=\mathbf{1 , 4 8 0}\end{array}$ & P-value & $\begin{array}{l}\text { Hispanics } \\
\mathrm{n}=\mathbf{1 , 4 3 5}\end{array}$ & P-value \\
\hline \multicolumn{6}{|l|}{$2012^{\dagger}$} \\
\hline In-hospital Mortality, \% & 4.95 & 3.85 & --- & 4.62 & --- \\
\hline Unadjusted OR (95\% CI) & Ref. & $0.75(0.18 ; 3.22)$ & .72 & $0.86(0.28 ; 2.57)$ & .90 \\
\hline Adjusted $O R^{*}(95 \%$ CI) & Ref. & $0.66(0.15 ; 2.94)$ & .59 & $0.98(0.33 ; 2.88)$ & .97 \\
\hline \multicolumn{6}{|l|}{2013} \\
\hline In-hospital Mortality, $\%$ & 4.58 & 4.55 & --- & 7.78 & --- \\
\hline Unadjusted OR (95\% CI) & Ref. & $0.99(0.35 ; 2.79)$ & .99 & $1.76(0.80 ; 3.87)$ & .16 \\
\hline Adjusted OR $^{*}(95 \%$ CI) & Ref. & $0.85(0.28 ; 2.53)$ & .77 & $1.77(0.75 ; 4.16)$ & .19 \\
\hline \multicolumn{6}{|l|}{2014} \\
\hline In-hospital Mortality, $\%$ & 3.59 & 3.21 & --- & 3.79 & --- \\
\hline Unadjusted OR (95\% CI) & Ref. & $0.89(0.36 ; 2.19)$ & .80 & $1.06(0.39 ; 2.89)$ & .91 \\
\hline Adjusted OR ${ }^{*}(95 \%$ CI) & Ref. & $0.58(0.23 ; 1.46)$ & .25 & $1.08(0.41 ; 2.86)$ & .88 \\
\hline \multicolumn{6}{|l|}{ Overall $(n=35,805)$} \\
\hline In-hospital Mortality, \% & 4.17 & 3.72 & --- & 5.23 & --- \\
\hline Unadjusted OR (95\% CI) & Ref. & $0.89(0.48 ; 1.64)$ & .70 & $1.27(0.74 ; 2.17)$ & .39 \\
\hline Adjusted OR ${ }^{t^{t}}(95 \%$ CI) & Ref. & $0.70(0.37 ; 1.32)$ & .27 & $1.34(0.79 ; 2.28)$ & .28 \\
\hline
\end{tabular}

Abbreviations: CI, confidence intervals; AA, African Americans; OR, odds ratios.

Note:Weighted data were used for all analyses.

${ }^{\dagger}$ A total of 465 patients were not included in the analysis due to one additional stratum that was eliminated from the adjustedmodel as it did not contain members of the subpopulation.

${ }^{*}$ Adjusted for demographics (i.e., age, gender), known coronary artery disease, prior percutaneous coronary intervention, priorcoronary artery bypass graft, and certain comorbidities (i.e., diabetes mellitus, renal failure, coagulopathy, fluid and electrolyte disorder, coronary heart failure, hypertension, pulmonary circulation disorder, depression, and cancer,)primary payer, median household income, hospital region, discharge dispositionand TAVR access site. 
Table 3.

Odds ratios of secondary in hospital outcomes among patients undergoing TAVR according to race/ethnicity.

\begin{tabular}{|c|c|c|c|c|c|c|}
\hline Outcomes & $\begin{array}{c}\text { Overall } \\
\mathbf{n}=\mathbf{3 6 , 2 7 0}\end{array}$ & $\begin{array}{c}\text { Non-Hispanic Whites } \\
\mathbf{n}=\mathbf{3 3 , 3 5 5}\end{array}$ & $\begin{array}{c}\text { African Americans } \\
\mathbf{n}=\mathbf{1 , 4 8 0}\end{array}$ & P-value & $\begin{array}{l}\text { Hispanics } \\
\mathrm{n}=\mathbf{1 , 4 3 5}\end{array}$ & P-value \\
\hline \multicolumn{7}{|l|}{ MACE } \\
\hline$\%$ & 8.48 & 8.27 & 10.47 & --- & 11.15 & --- \\
\hline Unadjusted & --- & Ref. & $1.30(0.87 ; 1.94)$ & .20 & $1.39(0.94 ; 2.05)$ & .10 \\
\hline \multicolumn{7}{|l|}{ OR $(95 \% C I)$} \\
\hline Adjusted & --- & Ref. & $1.14(0.76 ; 1.73)$ & .53 & $1.46(0.99 ; 2.15)$ & .05 \\
\hline \multicolumn{7}{|l|}{ OR $(95 \% C I)^{\dagger}$} \\
\hline \multicolumn{7}{|l|}{ NACE } \\
\hline$\%$ & 12.96 & 12.79 & 14.53 & --- & 15.33 & --- \\
\hline Unadjusted & --- & Ref. & $1.16(0.82 ; 1.64)$ & .40 & $1.24(0.88 ; 1.73)$ & .22 \\
\hline \multicolumn{7}{|l|}{ OR $(95 \% C I)$} \\
\hline Adjusted & --- & Ref. & $1.03(0.72 ; 1.46)$ & .89 & $1.28(0.91 ; 1.81)$ & .15 \\
\hline \multicolumn{7}{|l|}{ OR $(95 \% C I)^{\dagger}$} \\
\hline \multicolumn{7}{|l|}{ Stroke/TIA } \\
\hline$\%$ & 2.9 & 2.79 & 4.05 & --- & 4.53 & --- \\
\hline Unadjusted & --- & Ref. & $1.47(0.81 ; 2.67)$ & .20 & $1.65(0.96 ; 2.85)$ & .07 \\
\hline \multicolumn{7}{|l|}{ OR $(95 \% C I)$} \\
\hline Adjusted & --- & Ref. & $1.50(0.82 ; 2.74)$ & .19 & $1.81(1.04 ; 3.14)$ & .04 \\
\hline \multicolumn{7}{|l|}{ OR $(95 \% C I)^{\dagger}$} \\
\hline \multicolumn{7}{|l|}{ AMI } \\
\hline$\%$ & 2.2 & 2.05 & 3.38 & --- & 4.18 & --- \\
\hline Unadjusted & --- & Ref. & $1.67(0.86 ; 3.23)$ & .13 & $2.08(1.09 ; 3.97)$ & .03 \\
\hline \multicolumn{7}{|l|}{ OR $(95 \% C I)$} \\
\hline Adjusted & --- & Ref. & $1.39(0.70 ; 2.74)$ & .34 & $2.02(1.06 ; 3.85)$ & .03 \\
\hline \multicolumn{7}{|l|}{ OR $(95 \% C I)^{\dagger}$} \\
\hline \multicolumn{7}{|l|}{ PPM placement } \\
\hline$\%$ & 24.30 & 24.46 & 20.95 & --- & 24.04 & --- \\
\hline Unadjusted & --- & Ref. & $0.82(0.61 ; 1.10)$ & .19 & $0.98(0.73 ; 1.31)$ & .88 \\
\hline \multicolumn{7}{|l|}{ OR $(95 \% C I)$} \\
\hline Adjusted & --- & Ref. & $0.81(0.60 ; 1.09)$ & .17 & $0.99(0.74 ; 1.32)$ & .93 \\
\hline \multicolumn{7}{|l|}{ OR $(95 \% C I)^{\dagger}$} \\
\hline \multicolumn{7}{|c|}{ Conversion to SAVR } \\
\hline$\%$ & 0.43 & 0.45 & 0.0 & --- & 0.35 & --- \\
\hline Unadjusted & --- & Ref. & --- & --- & $0.77(0.11 ; 5.55)$ & .80 \\
\hline \multicolumn{7}{|l|}{ OR $(95 \% C I)$} \\
\hline Adjusted & --- & Ref. & --- & --- & $0.76(0.10 ; 5.80)$ & .79 \\
\hline
\end{tabular}




\begin{tabular}{|c|c|c|c|c|c|c|}
\hline Outcomes & $\underset{\mathbf{n}=\mathbf{3 6 , 2 7 0}}{\text { Overall }}$ & $\begin{array}{c}\text { Non-Hispanic Whites } \\
\mathbf{n}=\mathbf{3 3 , 3 5 5}\end{array}$ & $\begin{array}{c}\text { African Americans } \\
n=1,480\end{array}$ & P-value & $\underset{\mathrm{n}=1, \mathbf{4 3 5}}{\operatorname{Hispanics}}$ & P-value \\
\hline$\%$ & 18.6 & 18.06 & 21.62 & --- & 27.18 & --- \\
\hline Unadjusted & --- & Ref. & $1.25(0.94 ; 1.66)$ & .12 & $1.69(1.29 ; 2.22)$ & $<.01$ \\
\hline \multicolumn{7}{|l|}{ OR $(95 \% C I)$} \\
\hline Adjusted & --- & Ref. & $0.89(0.65 ; 1.23)$ & .49 & $1.65(1.23 ; 2.21)$ & $<.01$ \\
\hline \multicolumn{7}{|l|}{ OR $(95 \% C I)^{\dagger}$} \\
\hline \multicolumn{7}{|l|}{ Cardiogenic shock } \\
\hline$\%$ & 4.1 & 4.03 & 4.73 & --- & 5.23 & --- \\
\hline Unadjusted & --- & Ref. & $1.18(0.69 ; 2.03)$ & .55 & $1.31(0.77 ; 2.23)$ & .32 \\
\hline \multicolumn{7}{|l|}{ OR $(95 \% C I)$} \\
\hline Adjusted & --- & Ref. & $0.91(0.53 ; 1.57)$ & .73 & $1.28(0.74 ; 2.22)$ & .37 \\
\hline \multicolumn{7}{|l|}{ OR $(95 \% C I)^{\dagger}$} \\
\hline \multicolumn{7}{|l|}{ Cardiac arrest } \\
\hline$\%$ & 3.3 & 3.24 & 3.72 & --- & 5.23 & --- \\
\hline Unadjusted & --- & Ref. & $1.15(0.63 ; 2.10)$ & .64 & $1.65(0.97 ; 2.80)$ & .07 \\
\hline \multicolumn{7}{|l|}{ OR $(95 \% C I)$} \\
\hline Adjusted & --- & Ref. & $0.94(0.50 ; 1.74)$ & .84 & $1.61(0.95 ; 2.73)$ & .08 \\
\hline \multicolumn{7}{|l|}{ OR $(95 \% C I)^{\dagger}$} \\
\hline \multicolumn{7}{|l|}{ Major bleeding } \\
\hline$\%$ & 3.0 & 3.01 & 3.04 & --- & 2.44 & --- \\
\hline Unadjusted & --- & Ref. & $1.01(0.52 ; 1.96)$ & .98 & $0.80(0.37 ; 1.73)$ & .58 \\
\hline \multicolumn{7}{|l|}{ OR $(95 \% C I)$} \\
\hline Adjusted & --- & Ref. & $0.80(0.42 ; 1.54)$ & .51 & $0.74(0.33 ; 1.67)$ & .47 \\
\hline \multicolumn{7}{|l|}{ OR $(95 \% C I)^{\dagger^{\dagger}}$} \\
\hline \multicolumn{7}{|c|}{ Vascular complications } \\
\hline$\%$ & 3.2 & 3.12 & 3.38 & --- & 4.18 & --- \\
\hline Unadjusted & --- & Ref. & $1.09(0.58 ; 2.04)$ & .80 & $1.36(0.75 ; 2.46)$ & .32 \\
\hline \multicolumn{7}{|l|}{ OR $(95 \% C I)$} \\
\hline Adjusted & --- & Ref. & $1.06(0.55 ; 2.03)$ & .87 & $1.51(0.84 ; 2.72)$ & .17 \\
\hline \multicolumn{7}{|l|}{ OR $(95 \% C I)^{\dagger}$} \\
\hline \multicolumn{7}{|l|}{ Sepsis } \\
\hline$\%$ & 2.6 & 2.38 & 3.38 & --- & 5.57 & --- \\
\hline Unadjusted & --- & Ref. & $1.43(0.71 ; 2.88)$ & .31 & $2.42(1.41 ; 4.14)$ & $<.01$ \\
\hline \multicolumn{7}{|l|}{ OR $(95 \% C I)$} \\
\hline Adjusted & --- & Ref. & $1.00(0.46 ; 2.14)$ & .99 & $2.34(1.30 ; 4.22)$ & $<.01$ \\
\hline \multicolumn{7}{|l|}{ OR $(95 \% C I)^{\dagger}$} \\
\hline \multicolumn{7}{|l|}{ Length of Stay ${ }^{*+*}$} \\
\hline \multicolumn{6}{|l|}{ days } & --- \\
\hline Unadjusted & --- & Ref. & $1.12(1.00 ; 1.26)$ & .05 & $1.21(1.09 ; 1.35)$ & $<.01$ \\
\hline
\end{tabular}




\begin{tabular}{|c|c|c|c|c|c|c|}
\hline Outcomes & $\underset{\mathbf{n}=\mathbf{3 6}, 270}{\text { Overall }}$ & $\begin{array}{c}\text { Non-Hispanic Whites } \\
\mathbf{n}=\mathbf{3 3 , 3 5 5}\end{array}$ & $\begin{array}{c}\text { African Americans } \\
n=1,480\end{array}$ & P-value & $\begin{array}{l}\text { Hispanics } \\
\mathrm{n}=\mathbf{1 , 4 3 5}\end{array}$ & P-value \\
\hline \multicolumn{7}{|l|}{ OR $(95 \% C I)$} \\
\hline Adjusted & --- & Ref. & $0.99(0.89 ; 1.10)$ & .87 & $1.18(1.08 ; 1.29)$ & $<.01$ \\
\hline \multicolumn{7}{|l|}{ OR $(95 \% C I)^{\dagger}$} \\
\hline \multicolumn{7}{|c|}{ Average Hospital Cost ${ }^{t}$} \\
\hline Median (IQR), & 196,897 & 194,954 & 200,373 & --- & 251,355 & --- \\
\hline days & $(145,428 ; 272,908)$ & $(144,120 ; 270,490)$ & $(149,123 ; 273,066)$ & & $(189,508 ; 347,738)$ & \\
\hline Unadjusted & --- & Ref. & $1.02(0.95 ; 1.10)$ & .61 & $1.30(1.18 ; 1.42)$ & $<.01$ \\
\hline \multicolumn{7}{|l|}{ OR $(95 \% C I)$} \\
\hline Adjusted & --- & Ref. & $0.97(0.91 ; 1.03)$ & .31 & $1.27(1.18 ; 1.36)$ & $<.01$ \\
\hline OR $(95 \% C I)^{\dagger}$ & & & & & & \\
\hline
\end{tabular}

Abbreviations: CI, confidence intervals; OR, odds ratios; MACE, Major Adverse Cardiovascular Events (i.e., composite of all-cause mortality, myocardial infarction and stroke/TIA); NACE, Net Adverse Cardiovascular Events (i.e., composite of MACE, major bleeding and vascular complication); TIA, Transient Ischemic Attack; AMI, Acute Myocardial Infarction; PPM, Permanent Pacemaker; SAVR, surgical aortic valve replacement; AKI, Acute Kidney Injury

Note: Weighted data were used for all analyses.

*ength of stay was reported in patients discharged alive.

${ }^{*}$ Negative Binomial Regression Model was performed to determine differences between racial/ethnic groups; Pearson and Deviance statistics were performed to assess overdispersion of the data.

${ }^{\dagger}$ Adjusted for demographics (i.e., age, gender), known coronary artery disease, prior percutaneous coronary intervention, prior coronary artery bypass graft, and certain comorbidities (i.e., diabetes mellitus, renal failure, coagulopathy, fluid and electrolyte disorder, coronary heart failure, hypertension, pulmonary circulation disorder, depression, and cancer), primary payer, median household income, hospital region, discharge dispositionand TAVR access site. 